\title{
Health, Labour Productivity and Growth
}

\author{
Joan Muysken* \\ I. Hakan Yetkiner ${ }^{*}$ \\ Thomas Ziesemer*
}

\begin{abstract}
Under the standard neo-classical growth framework, conditional convergence studies assume that a country with a higher initial human capital among others 'performs' better. Nevertheless the growth implications of health, another component of human capital, compared to education, have not been investigated thoroughly within the optimum growth framework yet. The aim of this study is to show rigorously the positive association between per capita income and health status of an economy and thereby provide a theoretical background for using 'health' variables in conditional convergence analyses. This positive relationship between health and per capita output is first shown in the standard neo-classical growth framework where the health status is exogenously given. Endogenising health then enables us to analyse the impact of optimal expenditure on health care on steady state growth and transition dynamics.
\end{abstract}

Keywords: Cass-Koopmans growth models, health.

JEL Classification: O4.

\footnotetext{
${ }^{*}$ Department of Economics and MERIT, University of Maastricht, Maastricht, The Netherlands.

* Corresponding Author: I. Hakan Yetkiner Department of Economics, University of Groningen, Groningen, The Netherlands. Phone: +31-50-3637204. Fax: +31-50-3637337. E-mail: i.h.yetkiner@eco.rug.nl. We would like to thank Gerard H. Kuper for helpful comments. I. Hakan Yetkiner is grateful to the Turkish Academy of Sciences for providing financial assistance while he was a member of the Department of Economics, Middle East Technical University, Ankara, Turkey.
} 


\section{Introduction}

A key property of the neo-classical growth model is that an economy that starts out further below its own steady-state position tends to grow proportionately faster. The key word, however, is "own", for empirical studies showed that this so-called absolute catch up proposition clearly failed in terms of the cross-country data. Many studies —for instance Barro (1991), Barro and Sala-i-Martin (1992) and Mankiw, Romer, and Weil (1992) — have shown that so-called conditional convergence is empirically more successful. In these studies country-specific characteristics are taken into account to control for differences in steady states. A typical example is human capital in the form of education (for example, average years of schooling and literacy rate), which has consistently been used as a control variable in these studies.

Schultz (1961) and Mushkin (1962) have shown long time ago that human capital can also be accumulated through improvements in health. ${ }^{1}$ In this context it is surprising that the second component of human capital, health, has been largely ignored in the growth literature. Indicators of health status like life expectancy at birth and infant mortality rate have relatively rarely been used in convergence studies - see Barro and Sala-i-Martin (1995). Knowles and Owen (1995, 1997) introduced this in the growth literature. For example, in their 1995 paper they augmented Mankiw, Romer, and Weil's (1992) work by controlling for the health and education components of human capital separately. The theoretical part of their study takes the positive relation between output and health as given - as we do below. The authors then estimate this relation in a Solovian growth framework. Thus, contrary to our approach, optimal health expenditure is not considered.

This is not surprising, since the neglect of health as a relevant variable for economic growth is also encountered on the theoretical side. While the relationship between growth and education has been intensively investigated - see the many studies inspired by Lucas (1988) — the link between health and growth has hardly been researched in the theoretical literature. On the other hand it has long been conceived that health by its very nature has important implications on labour supply — see Mushkin (1962). This notion is taken up by Cuddington et al. (1994) who analyse long-term growth in the presence of a communicable disease, namely AIDS, under the assumption of exogenous health expenditure. They show that an epidemic 
disease has important implications for size, structure, and productivity of labour and therefore for the growth performance of an economy — see Bloom and Mahal (1992) for an opposite view specific to AIDS on empirical grounds. Again, optimal health expenditure is not considered. Moreover, our model, unlike Cuddington et al. (1994), is not specific to a certain disease and, in that sense, is a general health-growth model.

Another theoretical study is van Zon and Muysken (1997). They include health into the Lucas's (1988) endogenous growth framework. In their model healthy labour is not only used in the production of goods and knowledge, but it is also necessary to maintain health. As a consequence the characteristics of the health sector have a clear impact on economic growth and optimal health expenditures are analysed. Our model differs from van Zon and Muysken's 1997 model because their model is very hard to characterise in steady state situation due to the fact that there does not exist a closed-form solution of the model and the transitional dynamics are not available.

Against this background, the aim of this study is to show the association between the optimal health expenditure and status of an economy and all other variables. We thereby provide a theoretical background for using 'health' variables in conditional convergence analyses, starting from the labour productivity implications of health. To this end we introduce health in a standard Ramsey-type growth model. In that context we develop an alternative measure of health status of an economy: the ratio of man-hours effectively supplied (and employed) to the total amount of manhours available.

In section two, the basic model is presented. This model shows a positive contribution of good health to steady state output (and economic growth) for an exogenous health status. This exogeneity, however, can only be a first approximation. Therefore the model is extended in the third section to endogenise the health status, since assets have to be put aside to maintain and improve health. Consumers include this in their dynamic consumption-asset accumulation trade-off. Thus, the representative household's health optimisation problem is embodied in an optimal growth framework, which enables one to analyse the impact of changes in the expenditure of health care on steady-state growth and transition dynamics. An interesting finding of the study is that the optimal health expenditure and consumption

\footnotetext{
${ }^{1}$ This point has been brought to our attention by Knowles and Owen (1995; 1997).
} 
in the transition to the steady state are below (above) their steady state values if the ratio of the stocks of capital and health is below (above) its steady state value. In other words, if physical capital relative to health is relatively scarce (abundant) compared to the steady state values, optimal expenditures for health and consumption are lower (higher) than in the steady state but increase (decrease). The last section concludes and summarises the study.

\section{The Model}

This study builds on the standard Ramsey-type growth model — see Cass (1965) and Koopmans (1965). A typical assumption in standard neo-classical growth models is that each worker supplies a fixed amount of labour services per unit of time. By starting from labour supply implications of health, we will show how the performance of an economy is related to the health status of that economy.

\subsection{The Household ${ }^{2}$}

Assume a representative household consisting of $N$ members. It maximises overall utility, $U$, as given by

$$
U=\int_{0}^{\infty} \frac{c^{1-\theta}-1}{1-\theta} e^{n t} e^{-\rho t} d t \quad \theta, \rho>0
$$

In (1) $c$ is the quantity of consumption per person, $n$ is the (net) exogenous growth rate of the household members, and $\theta$ and $\rho$ are the elasticity of marginal utility and subjective rate of time preference, respectively. Let us assume that each member's labour supply, $l^{i}$, is a function of his/her health status in the form

$$
l^{i}\left(h^{i}\right)=\left\{\begin{array}{ll}
1 & h^{i}=1 \\
0 & h^{i}=0
\end{array} \quad i=1,2, \ldots \ldots, N\right.
$$

\footnotetext{
${ }^{2}$ We suppressed the time arguments for simplicity.
} 
In (2) $h^{i}$ denotes the health status of $i$. We assume that household members are either healthy or unfit to work, which corresponds to the values $h^{i}=1$ and $h^{i}=0$, respectively. Those who are unhealthy do not work and therefore they are not included in labour supply at any instant of time. So effective labour supply is the sum of labour supply of healthy workers. Suppose that there are $N_{l}$ healthy workers at a given time and $N_{l}<N$. As each healthy worker supplies inelastically one unit of labour, total effective labour supply is also $N_{l}$.

The health status of the economy can be approximated by its average health status. In our model, the average health is the sum of 'healthy persons', $N_{l}$, divided by population $N$. Thus, the health index of the economy is

$$
h=\frac{\sum_{i=1}^{N} h^{i}}{N}=\frac{N_{1}}{N}
$$

Equation (3) can also be read as the ratio of healthy man-hours to total man-hours available in an economy at any instant of time. Hence, by using the intuition behind equation (2), we express the health status of the economy in a convenient way. ${ }^{3} \mathrm{We}$ conjecture that our health status measure does fit better in a growth framework owing to the fact that life expectancy at birth and infant mortality rate reflect nutrition and many other components of social development as much as health. ${ }^{4}$

Let us assume for the moment being that $h=N_{l} / N$ is constant, which implies that population and healthy workers grow at the same (exogenous) rate $n$. We will relax this assumption in the following section by endogenising $h$.

The flow budget constraint for the household is

$\dot{A}=w N_{1}+r A-c N_{1}-c\left(N-N_{1}\right)$

In (4) $N-N_{l}$ is the number of sick household members, $A$ is the level of assets, and $w$ and $r$ are market-determined factor prices. According to equation (4), those who are sick are unable to work and, therefore, do not earn a wage income. Nevertheless, as is

\footnotetext{
${ }^{3}$ This approach is quite similar to that in van Zon and Muysken (1997) who also define productive labour as $h N$, where $h$ represents the health status.

${ }^{4}$ Our argument, nevertheless, does not mean that it is wrong to use these or other health status variables - see OECD (1999) for a rich set of health status variables.
} 
obvious from equation (4), sick members are supposed to keep on consuming (by spending savings and sharing current income at any combination). Therefore, the household's instantaneous utility function is independent of the health status of the household.

The flow budget constraint can be rewritten in per capita terms as follows:

$\dot{a}=w h+(r-n) a-c$

In (5) assets per person $a$ is simply $A / N$. The household's optimisation problem is to maximise the overall utility $U$ in equation (1), subject to the budget constraint in equation (5) given the stock of initial assets $a(0)$ and the transversality condition on the state variable $a$.

The present-value Hamiltonian is

$J=\frac{c^{1-\theta}-1}{1-\theta} e^{-(\rho-n) t}+\lambda\{w h+(r-n) a-c\}$.

The first-order conditions for a maximum of $U$ and the standard transversality condition imposed on assets per capita define the household's optimum, yielding ${ }^{5}$

$\frac{\dot{c}}{c}=\frac{1}{\theta}(r-\rho)$

Equation (7) is the 'standard' expression for the optimum growth rate.

\subsection{The Firm}

Suppose that there is perfect competition in the goods sector. A representative firm has the following production function

$Y=K^{\alpha} N_{1}^{1-\alpha} \quad 0<\alpha<1$ 
In (8) $K$ is the aggregate capital stock, and $N_{l}$ is the number of healthy workers. ${ }^{6}$ The per capita production function becomes

$y=k^{\alpha} h^{1-\alpha}$

In (9) $h=N_{l} / N$ as previously. The representative firm's flow of profit $\pi$ at any point is

$\pi=N\left\{k^{\alpha} h^{1-\alpha}-w h-(r+\delta) k\right\}$

In (10) $r+\delta$ is the effective cost of capital and $k=K / N$. The first-order conditions for profit maximisation then yield:

$r=\alpha k^{\alpha-1} h^{1-\alpha}-\delta$

$w=(1-\alpha) k^{\alpha} h^{-\alpha}$

The health status variable distinguishes equation (11a) and (11b) from the standard results.

\subsection{Market Equilibrium}

We consider a closed economy model with no government. The assets accumulated by the households are used to finance the stock of capital, that is the interest rate mechanism will ensure $a=k$. Then using the household's flow budget constraint given in equation (5) and the conditions for $r$ and $w$ in equations (11a) and (11b) we get

$\dot{k}=k^{\alpha} h^{1-\alpha}-(n+\delta) k-c$

\footnotetext{
${ }^{5}$ Since the utility function (1) satisfies the Inada (1963) conditions we know that consumption will always be a positive finite number.

${ }^{6}$ In Yetkiner et al. (1999) we argue that external effects in specification of production function becomes crucial when the public provision of health is considered, which falls out of aims of this study.
} 
Moreover, substituting the interest rate in the solution of the household's optimisation problem —cf. equation (7)— yields

$\frac{\dot{c}}{c}=\frac{1}{\theta}\left(\alpha k^{\alpha-1} h^{1-\alpha}-\rho-\delta\right)$

Equations (12) and (13) construe the equations of motion in $c$ and $k$.

The constant steady-state values for per capita consumption $c$ and per capita capital stock $k$ are determined by setting the expressions in equations (12) and (13) equal to zero:

$$
\begin{aligned}
& \bar{k}=h\left(\frac{\alpha}{\rho+\delta}\right)^{\frac{1}{1-\alpha}} \\
& \bar{c}=h\left(\frac{\rho+\delta}{\alpha}-(n+\delta)\left(\frac{\alpha}{\rho+\delta}\right)^{\frac{1}{1-\alpha}}\right.
\end{aligned}
$$

In (14) a bar on top of a variable denotes steady state. Note that the standard perfecthealth Ramsey model's results are obtained when $h=1$. With $h$ smaller than unity, steady-state values of macroeconomic variables $k, y$, and $c$ are lower than the respective standard perfect-health results. Figure 1 below compares these two cases where ' $p h$ ' stands for 'perfect health' Ramsey results in the figure. The arrows of motion indicate the saddle-point stability of the steady state.

\section{Insert Figure 1 here}

The steady-state analysis shows equilibrium values of $c$ and $k$ (and thus $y$ ) lower than in the perfect-health Ramsey model. In Figure 1 an exogenous increase in the health status of the economy - represented by an increase in $h-$ shifts the $\dot{c}=0$ line to the right and moves the $\dot{k}=0$ curve up. ${ }^{7}$ These shifts generate increases in $\bar{c}, \bar{k}$ and $\bar{y}$. This suggests that, in terms of its effects on growth, a change in the average health level of the population works in the same way as an exogenous change in the level of

\footnotetext{
${ }^{7}$ For example, any exogenous development in curative or preventive medical technology may be the source of that shift.
} 
productivity in the Solow model. The crucial difference with productivity is that health only has an upper limit, namely perfect health.

Our findings provide a theoretical background for using health status variables to characterise countries in conditional convergence analysis. A convergence analysis shows that the constant $h$ does not appear in the $\beta$-convergence coefficient - see Barro and Sala-i-Martin $(1992 ; 1995)$ — for the reason that health status is assumed to be constant. Therefore $h$ behaves as if an exogenous productivity parameter, which is not against the neo-classical conditional convergence argument. It is worth noting that $h$ is neither constant nor exogenous in reality. In fact, health status and income affect each other. ${ }^{8}$ Therefore, in the next section, we will endogenise the health measure to enrich our theoretical inquiry into the relationship between health status and per capita output and growth.

\section{Endogenous Health and Growth}

This section extends the previous analysis by assuming that the health status of the economy is endogenously determined within the model by allowing representative household to optimise her health status in the consumption asset-accumulation tradeoff. We first elaborate the specification of the production function of the health status accumulation. Next, we discuss the representative household's trade-off when health status is endogenised and also elaborate the impact on market equilibrium. Finally we analyse the implications for the relation between health and growth in the model at steady state.

\subsection{Endogenous Health}

As mentioned above we now assume that the health status is no longer exogenous, but health expenditures have to be made to maintain and improve health. These expenditures can be preventive (e.g., hindering dissemination of diseases) and/or curative (aiming to regain some sick labour). Both kinds of health expenditures are

\footnotetext{
${ }^{8}$ As an example, look at Carrin and Politi (1995) to see the 'concave' relationship between life expectancy and real GDP per capita and the 'rectangular hyperbolic' relationship between infant mortality rate and real GDP per capita for 1990. Causality tests between life expectancy and real GDP per capita for high, middle, and low income countries show that the direction of causality changes from
} 
inevitable, because otherwise participation of labour in the production process is subject to a constant decay of the healthy labour stock. Actually we assume that in the absence of health expenditures, the number of healthy workers decreases at a rate $v$. However, this implies that workers that become sick fall only out of labour market but they do not die. Therefore $v$ is neither the mortality rate nor does it have any contribution to that rate.

Since we assume $v>n$, the number of healthy workers will decrease at a rate $v-n$. The impact of health expenditures $X$ is to stop or slow down the constant decay of healthy labour and to bring the ratio of healthy labour to total labour, $h$, to some optimal level. We define the healthy workers' accumulation function as follows:

$$
\dot{N}_{1}=\zeta X^{\beta} N^{1-\beta}-(v-n) N_{1} \quad 0<\beta<1
$$

where we assume that aggregate health expenditures $X$ have decreasing returns, and $\zeta$ is a productivity parameter. ${ }^{9}$ The generation of healthy labour $N_{l}$ depends on the existing stock of labour $N$ as much as on preventive and curative health expenditures. This is so because health expenditures produce healthy labour from healthy labour (preventive effect) or from sick workers (curative effect). ${ }^{10}$

Since the Inada (1963) conditions hold for the production function (8), each factor of production is necessary for positive output. This implies that health expenditures are necessary and inevitable in this model. To see this, suppose for the moment that health expenditures are zero. Then, from equation (15), it is clear that the healthy labour stock would 'depreciate' at a constant rate. This would force (healthy) labour to zero at some point in time and thus output would be zero. Since the marginal return is very high as any factor of production approaches zero, the representative household would always prefer to incur some positive amount of health expenditures.

We can re-write equation (15) by defining preventive and curative health expenditures per person $x=X / N$ and using the relationship $\dot{h} / h=\dot{N}_{1} / N_{1}-\dot{N} / N$, which yields:

one to another as the income group changes, cf. Mazumdar (1996). Similar results are found for infant survival rate.

${ }^{9}$ van Zon and Muysken (1997) use a similar function, except that they use productive labour to counter this decay.

${ }^{10}$ Equation (15) is, in essence, a customary stock variable accumulation function frequently used in the growth literature See for example see Barro and Sala-i-Martin (1995). 


$$
\frac{\dot{h}}{h}=\zeta x^{\beta} h^{-1}-v
$$

This relation should be added as an additional constraint to the household optimisation process.

\subsection{The Household's Trade Off}

As a consequence of the necessity of health expenditures, households face a trade-off that endogenously determines the health status of an economy. On the one hand, by being healthier they participate more in the production process and therefore contribute positively to their welfare at any instant of time. On the other hand, they incur some health expenditures, which is foregone consumption, to maintain or improve their healthiness. This trade-off shows up in the intertemporal budget constraint, where health expenditures are at the detriment of asset accumulation:

$$
\dot{a}=w h+(r-n) a-c-x
$$

The constraints to maximisation of the utility function (1) are now not only the amended budget constraint (17), but also the healthy worker's accumulation function (16). This defines the dynamic optimisation process, in which households determine the optimal health status they would like to have.

Then the present value Hamiltonian becomes

$$
J=\frac{c^{1-\theta}-1}{1-\theta} e^{-(\rho-n) t}+\lambda\{w h+(r-n) a-c-x\}+\mu\left(\zeta x^{\beta} h^{-1}-v\right) h
$$

In (18) $c$ and $x$ are the choice variables, $a$ and $h$ are the state variables, and $\lambda$ and $\mu$ are the co-state variables. ${ }^{11}$

By solving equation (18) through the method of optimum control, one finds that the optimal path for consumption still is given by equation (7). This implies that

\footnotetext{
${ }^{11}$ See Appendix A for a complete solution of the optimum version of the model. Equations (11a,b) are still applicable because the market solution and that of the central planner are the same.
} 
as usual the interest rate determines consumption growth. The optimal path for health expenditures is given by

$\dot{x}=\frac{v+r-n}{1-\beta} x-\frac{\beta \zeta}{1-\beta} w x^{\beta}$

This should be considered simultaneous with the path for health creation (16) - we elaborate this in the next section.

\subsection{Market Equilibrium}

Although firm behaviour is not directly affected by expenditures on health, one should realise that health also affects marginal productivity of both labour and capital and hence both the interest rate and the wage rate, as can be seen from equations (11a) and (11b), respectively. Moreover, since the accumulation of assets is influenced by health expenditures, the accumulation of capital will be too. We will return to that later on. First we discuss the dynamic process between health expenditures and health creation.

Note that substitution of (11a) and (11b) in the equation of motion for health expenditures $x$, yields:

$\dot{x}=\frac{v-n+\alpha\left(\frac{h}{k}\right)^{1-\alpha}-\delta}{1-\beta} x-\frac{\beta \zeta(1-\alpha)}{1-\beta}\left(\frac{k}{h}\right)^{\alpha} x^{\beta}$

The dynamics between health expenditures and health creation then can be shown for any given value of the capital stock $k$, using equations (20) and (16), respectively. This is elaborated in Figure 2 where one sees that the $\dot{x}=0$ line is decreasing in $h$ and the $\dot{h}=0$ line is increasing. Moreover, the equations of motion show that this part of the system is saddle-point stable. The equilibrium values, as functions of $k$, then are: 
$h^{*}(k)=\frac{\zeta}{\mu}\left[\frac{\beta \zeta(1-\alpha)}{(v-\delta-n)\left(\frac{h^{*}(k)}{k}\right)^{\alpha}+\alpha \frac{h^{*}(k)}{k}}\right]^{\frac{\beta}{1-\beta}}$

and

$x *(k)=\left[\frac{v}{\zeta} h *(k)\right]^{\frac{1}{\beta}}$

Insert Figure 2 here

From the figure one also sees that the saddle-path shows a negative relation between health expenditures and health. This is plausible since optimal health expenditures will be relatively high when health is relatively bad and vice versa, because health expenditures are an investment in the production factor labour. ${ }^{12}$

A problem with this partial analysis is that a simultaneous movement of $k$ will shift the stationary line for $x$ and the saddle path of Figure 2. If, e.g., the movement goes from high to low $x$ and from low to high $h$, the steady state is approached from the left. However, a simultaneous increase in $k$, moving up the curves, reinforces the increase in $h$ but counteracts the decrease of $x$. This raises the question which of the two effects on $x$ is stronger.

Analogous to equation (12), the accumulation function of capital is found from the budget constraint given in equation (17) to be:

$\dot{k}=k^{\alpha} h^{1-\alpha}-(n+\delta) k-c-x$

A second problem is that the stationary lines of Figure 1 are now shifted by movements in $h$ and $x$. Thus, we have to deal with the interaction of the variables $c, k$, $h, x$ as captured in the equations (13), (16), (20) and (22). Although it is clear that our concave Hamiltonian function will result in a unique optimal growth path, it is far from clear what the dynamic process exactly looks like.

Theorems 5.3 and 5.4 in Feichtinger and Hartl (1986) provide conditions under which a linear approximation of a $4 \times 4$ system in connection with dynamic

\footnotetext{
${ }^{12}$ For simplicity we have neither $\mathrm{h}$ nor $\mathrm{x}$ as an argument in the utility function.
} 
optimisation - i.e. of the so-called canonical system - will have two positive and two negative real roots (see appendix B for details) ${ }^{13}$. In this case the constants of the two positive roots can be put equal to zero. Otherwise it would explode, which cannot be optimal. Consequently, the system for the analysis of local stability can be split up into two parts: First, the dynamics of $h$ and $k$ is considered separately; second, the dynamics of $k$ and $h$ is fed back into that of $c$ and $x$, both in a very simple way. This separation avoids the feed back of $c$ and $x$ into the $(k, h)$-system and makes the whole problem tractable.

Unfortunately, we can prove some of the conditions critical to the application of the theorem only numerically (see appendix $\mathrm{C}$ ). Here we focus on the results. The parameter values used so far are the following: We put the rate of capital depreciation at $\delta=0.03$ in accordance with national accounting results (see Mankiw, Romer and Weil 1992). The share of capital is assumed to be $\alpha=0.3$; the rate of time preference is set equal to $\rho=0.06$ in order to make sure that $y / k=0.3$ in accordance with equation (A.9). We use $n=0.01$ as in Barro and Sala-i-Martin 1995. Next we fix $v=0.02$ and $\beta=0.5$ which make sure that health expenditure as a share of GDP is about $10 \%$ in the steady state ${ }^{14}$ and consumption is between 70 and $80 \%, 76,67 \%$ for our values. Finally, we fix $\zeta=0.045$ which makes sure that $h<1$ in the steady state allowing us to avoid corner solutions for $h=1$. Using these values we show in appendix $\mathrm{C}$ that the conditions of the theorem hold and derive the lines for the stationary loci for h and $\mathrm{k}$ (see Figure 3).

Insert figure 3 over here

Both lines turn out to have positive slopes in $(h, k)$-space. The slope of the stationary line for $k$ is $15 \%$ and the slope of the line for $h$ is about $7 \%$. Arrows in Figure 3 indicate that the system is stable for any given initial values of $h$ and $k$. Hence $k$ and $h$ will always converge towards their steady state values. When the initial values of $k$ and $h$ are in region $\mathrm{B}, k / h$ will increase whereas in region $\mathrm{D}$ it will

\footnotetext{
${ }^{13}$ Readers non-familiar with German may have a look at Turnovsky (1981).

${ }^{14}$ The business press reports that health expenditures as a percentage of GDP are 6,7\% in the UK, $13,9 \%$ in the US and slightly below $10 \%$ in the Netherlands and Germany. Therefore fixing it at about $10 \%$ is a reasonable order of magnitude.
} 
decrease. In regions $\mathrm{A}$ and $\mathrm{C}$ the ratio $k / h$ can both increase and decrease when moving towards the steady state.

\section{INSERT FIGURE 4 OVER HERE}

The implications for consumption of the movement of $k / h$ towards the steady state follow from equation (13). This is drawn in Figure 4, putting $k / h$ on the horizontal and $c$ on the vertical axis. If $k / h$ starts below (above) its steady-state value and therefore increases (decreases), the change in $c$ must be positive (negative). By implication, the initial value of $c$ must be below (above) its steady-state value.

\section{INSERT FIGURE 5 OVER HERE}

The implications for health expenditures of the movement of $k / h$ toward the steady state follow from equation (20). Figure 5 reveals that in the neighbourhood of the steady state we have $\partial \dot{x} / \partial x>0$. This means that equation (20) is unstable for given values of $k / h$ and can only be stabilised by shifts of $k / h$. The stationary value of $x$ increases with the ratio $k / h{ }^{15}$ As an increase (decrease) of $\mathrm{k} / \mathrm{h}$ therefore shifts the stationary line to the right (left). If $k / h$ increases (decreases) on its way to the steady state according to Figure 3, $x$ must be to the right (left) of the stationary line and the change in $x$ must therefore be positive (negative) until it comes to a hold through the shift in the stationary line. In other word if $k / h$ is below (above) its steady-state value the value for optimal health expenditure $x$ is below (above) its steady-state value and increasing (decreasing). This is the same behaviour as that of optimal consumption. Therefore the optimal consumption and health expenditures depend on the relative values of $k / h$ in the transition relative to those in the steady state. Optimal health expenditure and consumption in the transition to the steady state are below (above) their steady-state values if the ratio of the stocks of capital and health is below (above) its steady-state value. In other words, if physical capital relative to health is relatively scarce (abundant) compared to the steady-state values, optimal expenditures

\footnotetext{
15 The stationary value of $x$ from equation (20) can be calculated as $x_{\mid \dot{x}=0}=\left[\frac{v-n-\delta+\alpha(k / h)^{\alpha-1}}{\beta \zeta(1-\alpha)(k / h)^{\alpha}}\right]^{\frac{1}{\beta-1}}$.
} As the exponent is negative, the stationary value of $x$ increases with $k / h$. 
for health and consumption are lower (higher) than in the steady state but increase (decrease).

\subsection{The Impact of Health Parameters}

It is interesting to analyse the impact of the characteristics of the health sector on the outcome of the model. The steady-state value of the health index is:

$\bar{h}=\left(\frac{\beta(1-\alpha)}{v+\rho-n}\left(\frac{\alpha}{(\rho+\delta)}\right)^{\frac{\alpha}{1-\alpha}}\right)^{\frac{\beta}{1-\beta}} \frac{\zeta^{\frac{1}{1-\beta}}}{v}$.

The steady-state health expenditures are:

$\bar{x}=\left(\frac{\beta \zeta(1-\alpha)}{\rho-n+v}\left(\frac{\alpha}{(\rho+\delta)}\right)^{\frac{\alpha}{1-\alpha}}\right)^{\frac{1}{1-\beta}}$

The health sector is characterised mainly by its productivity $\zeta$, the population growth rate $n$, and the rate of decay of health $v$. The impact of these parameters on the steady state of the model is summarised in Table 1.

Table 1 Impact of health parameters on the steady state

\begin{tabular}{|c|c|c|c|c|c|}
\hline Steady state value of & \multirow{2}{*}{$\mathrm{y}$} & \multirow[t]{2}{*}{$\mathrm{c}$} & \multirow[t]{2}{*}{$\mathrm{k}$} & \multirow[t]{2}{*}{$\mathrm{h}$} & \multirow[t]{2}{*}{$\mathrm{x}$} \\
\hline Parameters & & & & & \\
\hline$\zeta$ & + & + & + & + & + \\
\hline $\mathrm{n}$ & + & $?$ & + & + & + \\
\hline $\mathrm{V}$ & - & - & - & - & - \\
\hline
\end{tabular}

From the table one sees that an increase in productivity $\zeta$ affects all steady state variables in a positive way. It seems rather obvious that an increase in productivity in health care will lead to an improvement in health, cet. par. This will enhance both 
capital accumulation and consumption. Hence output and the capital stock will increase too. Finally, the increase of health expenditures follows from a higher marginal return from these expenditures in the trade-off with consumption and investment in physical capital.

The negative impact of an increased rate of decay $v$ on health is plausible because a higher rate directly means more sick workers. There also is an indirect effect through lower health expenditures, which result from a diminished marginal productivity of these expenditures. The negative effects on consumption, output and capital follow directly.

Finally, higher population growth $n$ has a positive effect on health expenditures because it increases their productivity. The effects on capital and output then follow directly. The impact on consumption is ambiguous, however, because, on the one hand, consumption is affected negatively by population growth in the perfect health situation (as in Cass-Koopmans). However, the steady-state value of $k$ also appears in the end of the expression for $c$. It depends on that of $h$ because health is directly affected positively as can be seen from (15). This produces a positive incentive to increase health expenditure as can be seen from (25). In sum, as in CassKoopmans the $c / k$ ratio is negatively related to population growth, but the optimal $c$ not necessarily decreases with population growth ${ }^{16}$.

\section{$4 \quad$ Concluding Remarks}

In this paper we have investigated optimal health expenditure and consumption by adding a health accumulation function to the Cass-Koopmans optimum-growth model.

The major finding was that optimal health expenditure and consumption in the transition to the steady state are below (above) their steady-state values if the ratio of the stocks of capital and health is below (above) its steady state value. In other words, if physical capital relative to health is relatively scarce (abundant) compared to the

\footnotetext{
${ }^{16}$ More technically, consumption is affected negatively by population growth in the perfect health situation (as in Cass-Koopmans) as can be seen also from the direct effects appearing in A.16. However, the steady-state value of $\mathrm{k}$ also appears in the end of the expression for $\mathrm{c}$. It depends on that of $\mathrm{h}$ (according to A.12) and that is positively affected by $\mathrm{n}$ as health expenditure was.
} 
steady state values, optimal expenditures for health and consumption are lower (higher) than in the steady state but increase (decrease).

This result was found with the help of a theorem that allows to separate the analysis of the dynamics of the state variables from that of the control variables. However, results could only be obtained for one set of parameter values for which the theorem could be applied. Other parameter values may lead to more complicate solutions. But so far we have no indication that such a set of parameter values can be found for reasonable orders of magnitude of the variables of the model. The search for other constellations is left for future research.

A second finding was that steady-state consumption is no longer necessarily negatively related to population growth (as it is in the standard model) because it enhances the steady-state percentage of health workers under the assumption of the health accumulation function used. An interesting alternative to this function is the epidemic health function used by Cuddington et al. (1994). However, none of the two functions is obviously better suited to modelling health processes then the other.

Finally, the limits that some readers may see for the Cass-Koopmans model are of course also limits of our analysis. One of these limits is the absence of endogenous growth. The transitional relation between health and technical change will be an interesting subject for future research. 
Figure 1 Comparison of equilibrium points in perfect health and imperfect health

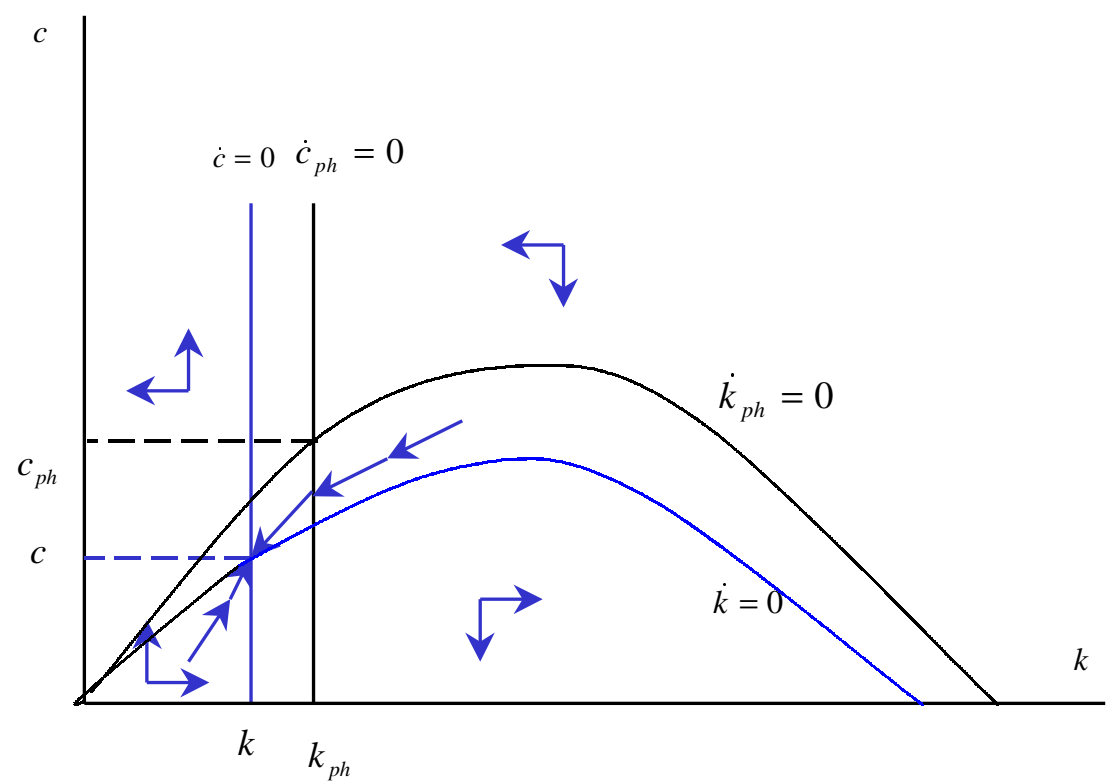

Figure 2 Stability of $x$ and $h$, conditional on $k$

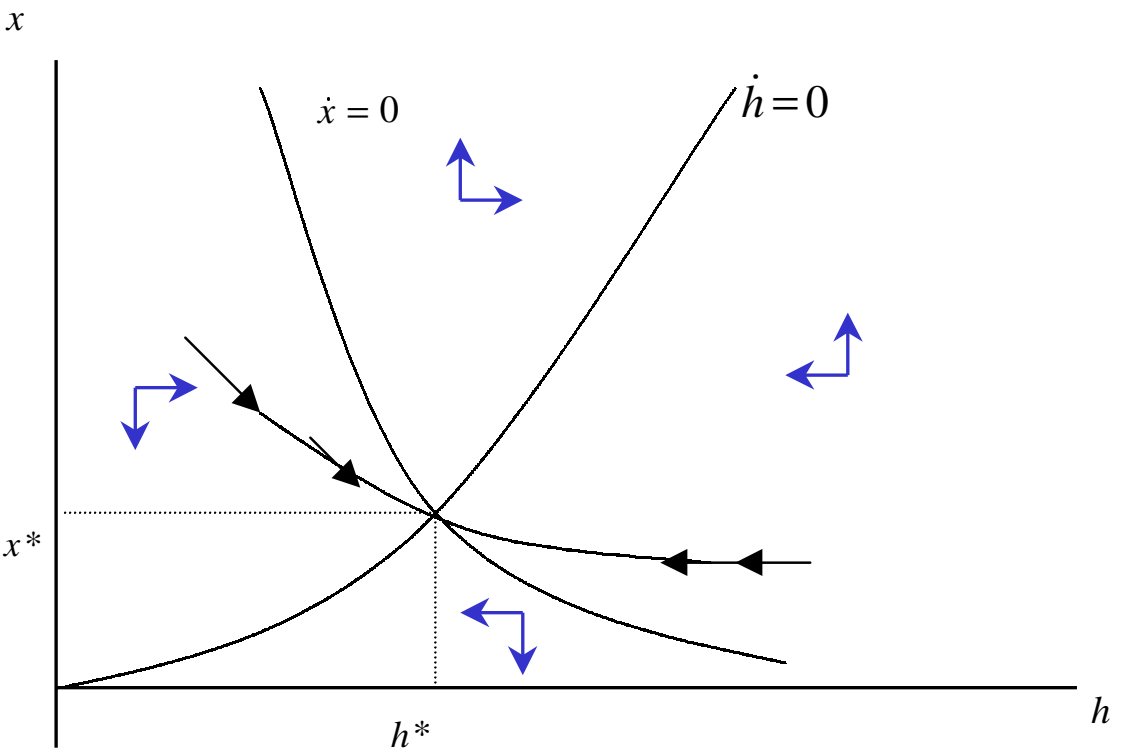


Figure 3 Local dynamics of the optimal health and capital stocks

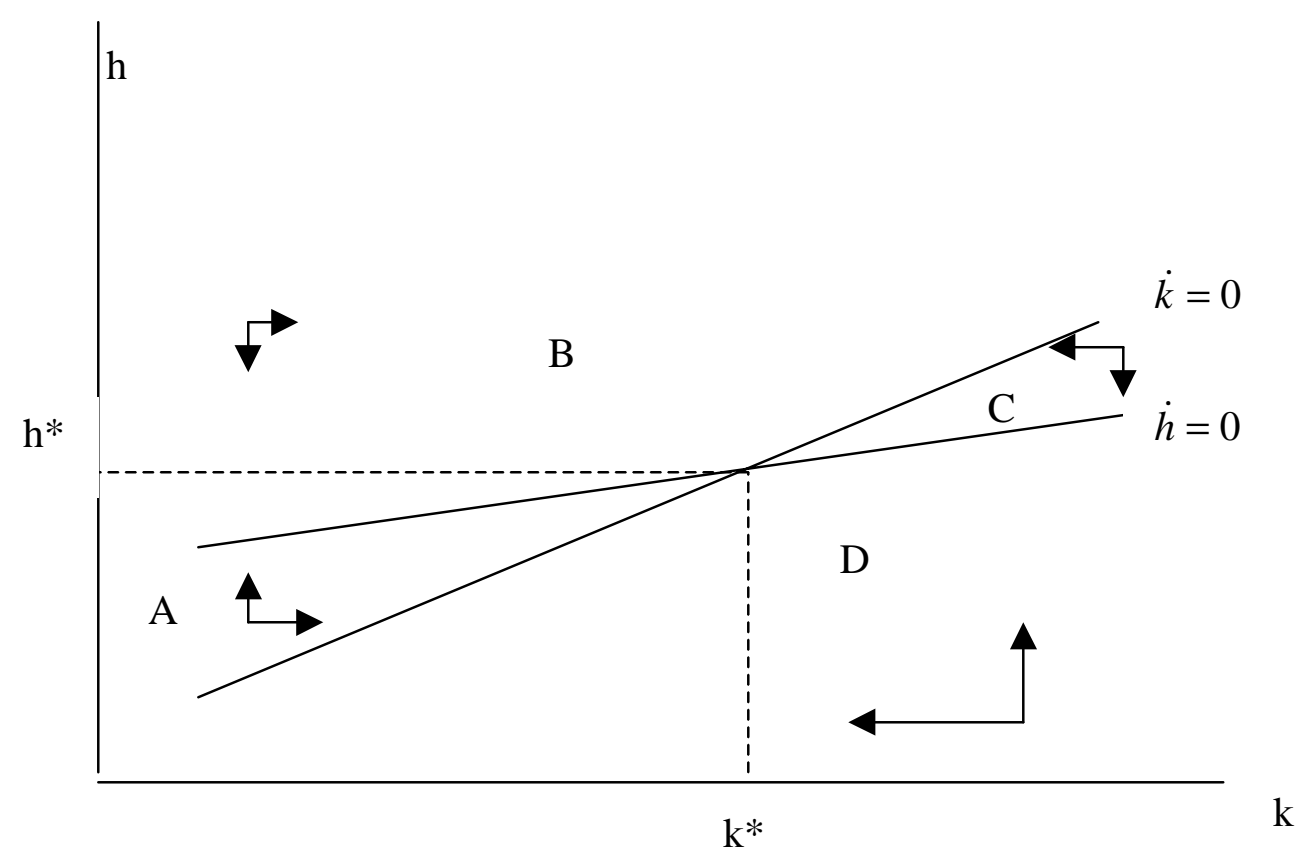

Figure 4 Capital-healthy labour ratio driving optimum consumption

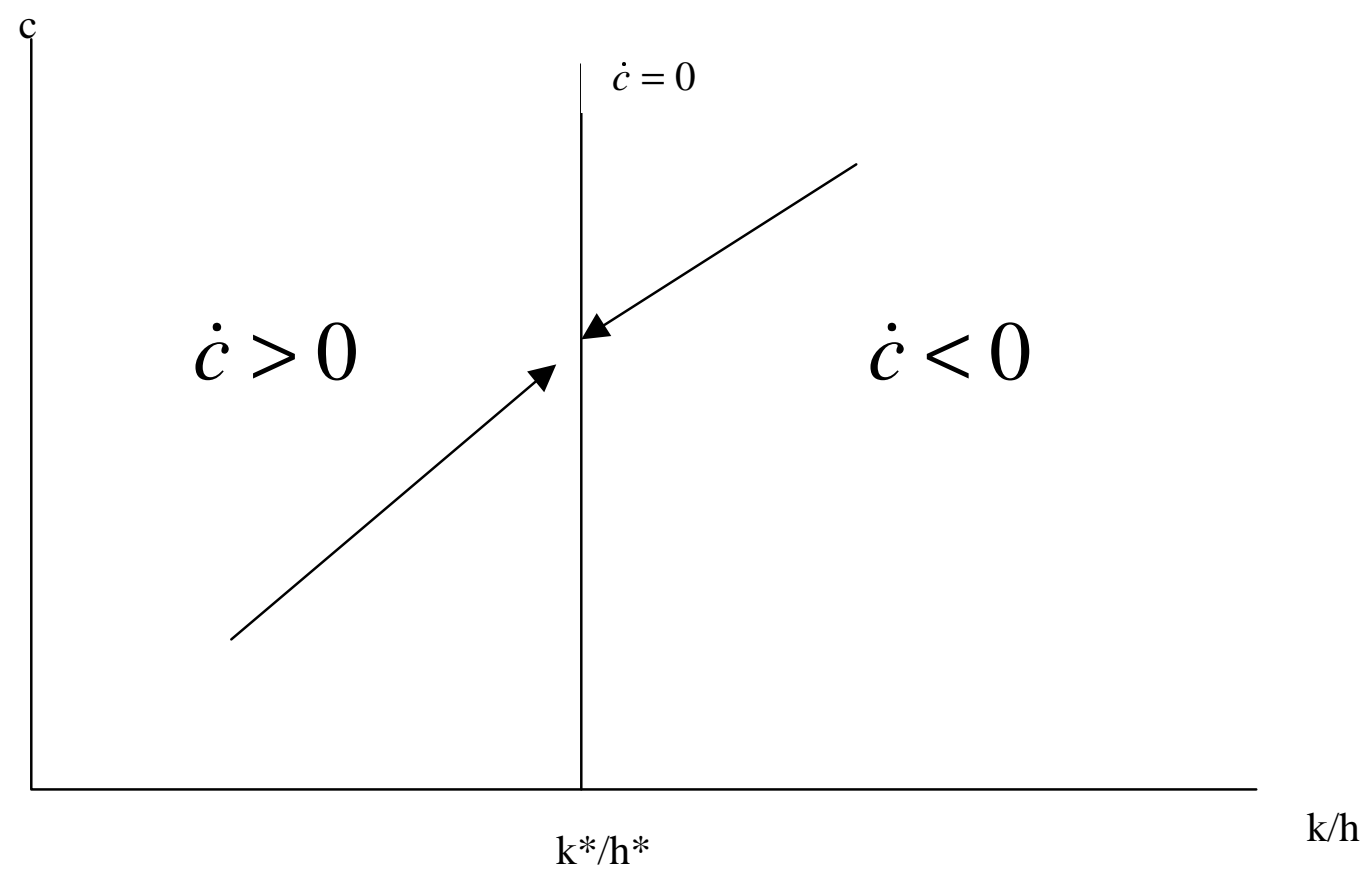


Figure $5 \quad$ Optimum health expenditure stabilised by capital and health growth

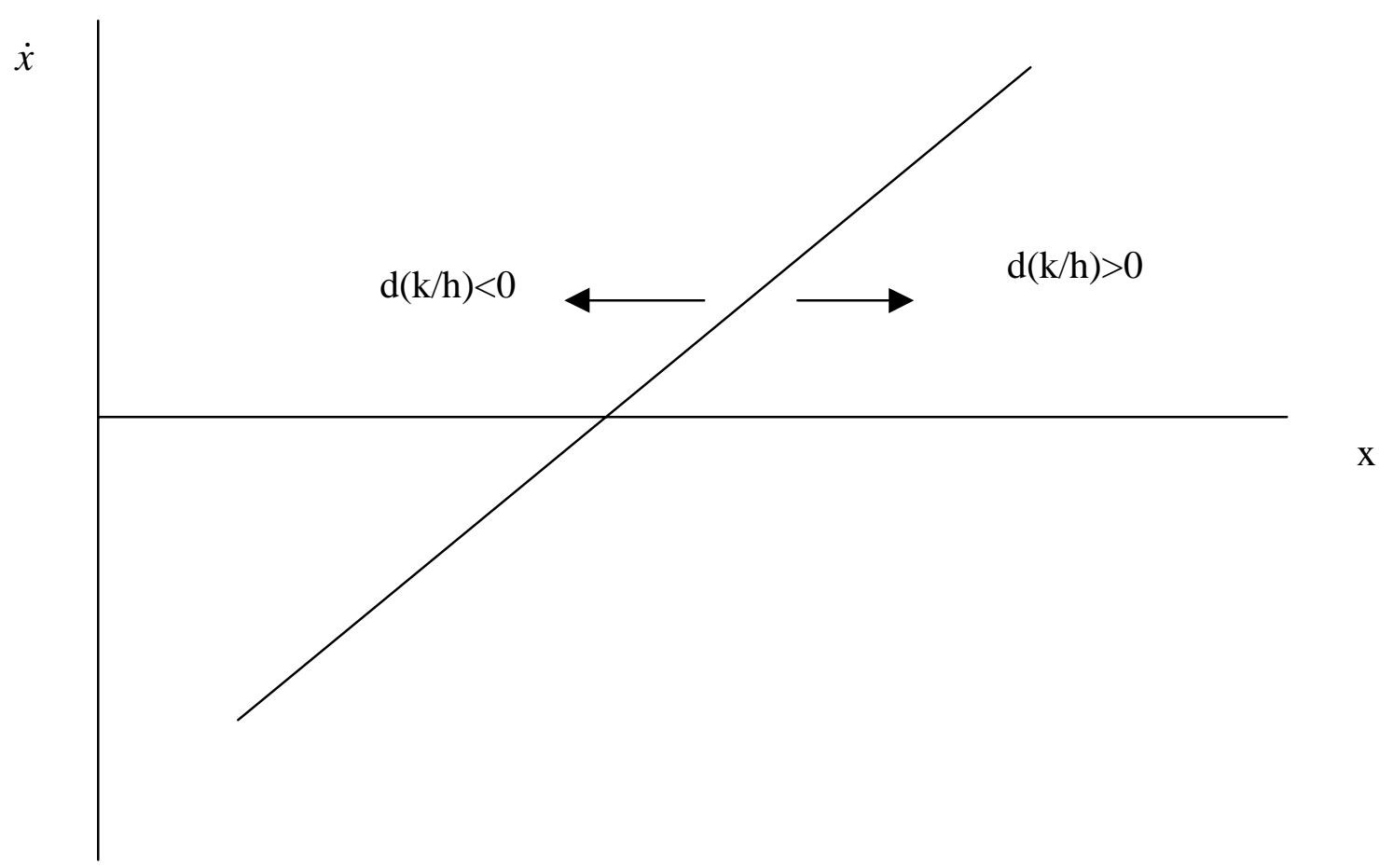




\section{Appendix A}

The current-value Hamiltonian for the central planner's problem is

$H=\frac{c^{1-\theta}-1}{1-\theta} e^{n t}+\lambda\left\{K^{\alpha} N_{1}^{1-\alpha}-c N-X-\delta K\right\}+\mu\left\{\zeta X^{\beta} N^{1-\beta}-(v-n) N_{1}\right\}$

$\lambda$ and $\mu$ are the co-state variables. The first-order conditions are following:

$$
\begin{aligned}
& \frac{\partial H}{\partial c}=c^{-\theta}-\lambda=0 \\
& \frac{\partial H}{\partial X}=-\lambda+\mu \zeta \beta x^{\beta-1}=0 \\
& -\frac{\partial H}{\partial K}=\dot{\lambda}-\rho \lambda=-\lambda\left(\alpha K^{\alpha-1} N_{1}^{1-\alpha}-\delta\right) \\
& -\frac{\partial H}{\partial N_{1}}=\dot{\mu}-\rho \mu=-\lambda K^{\alpha}(1-\alpha) N_{1}{ }^{-\alpha}+\mu(v-n)=-\lambda(k / h)^{\alpha}(1-\alpha)+\mu(v-n) \\
& \frac{\partial H}{\partial \lambda}=\dot{K}=K^{\alpha} N_{1}^{1-\alpha}-c N-X-\delta K \\
& \frac{\partial H}{\partial \mu}=\dot{N}_{1}=\zeta X^{\beta} N^{1-\beta}-(v-n) N_{1}
\end{aligned}
$$

Solving A.2 for $\mathrm{c}$ and A. 3 for $\mathrm{x}$ and using the definitions for $\mathrm{k}$ and $\mathrm{h}$ yields the canonical system:

$$
\begin{aligned}
& \dot{k}=k^{\alpha} h^{1-\alpha}-\lambda^{\frac{-1}{\theta}}-\left[\frac{\lambda}{\mu \beta \zeta}\right]^{\frac{1}{\beta-1}}-(n+\delta) k \\
& \dot{h}=\zeta\left[\frac{\lambda}{\mu \zeta \beta}\right]^{\frac{-\beta}{1-\beta}}-v h \\
& \dot{\lambda}=\lambda\left[\rho-\alpha(k / h)^{\alpha-1}+\delta\right] \\
& \dot{\mu}=\mu(v-n+\rho)-\lambda(1-\alpha)(k / h)^{\alpha}
\end{aligned}
$$




\section{STEADY-STATE SOLUTIONS}

In a situation of steady-state growth $\mathrm{k}, \mathrm{h}, \lambda$ and $\mu$ would have to be constant as would $\mathrm{c}$ and $\mathrm{x}$.

$\hat{y}=\hat{k}=\hat{h}=\hat{c}=\hat{x}=\hat{\lambda}=\hat{\mu}$

From $\left(A .4^{\prime}\right)$ we get

$\frac{\bar{y}}{\bar{k}}=\frac{\rho+\delta}{\alpha}$

Setting (A.7') equal to zero yields

$\frac{\lambda}{\mu}=\left[\frac{v h}{\zeta}\right]^{\frac{1-\beta}{-\beta}} \zeta \beta$

Setting (A.5') equal to zero yields (using A.9 in the second equation below)

$\overline{\left(\frac{\lambda}{\mu}\right)}=\frac{v-n+\rho}{(1-\alpha)(k / h)^{\alpha}}=\frac{v-n+\rho}{(1-\alpha)\left(\frac{\rho+\delta}{\alpha}\right)^{\frac{-\alpha}{1-\alpha}}}$

In order to get positive shadow prices the numerator must be positive. Equating (A.10) and (A.11) and solving for h yields (where $\bar{h} \leq 1$ by definition)

$\left.\bar{h}=\left[\frac{v-n+\rho}{\zeta \beta(1-\alpha)\left(\frac{\rho+\delta}{\alpha}\right)^{\frac{-\alpha}{1-\alpha}}}\right]^{\frac{\zeta}{v}<1}\right]^{\frac{-\beta}{1-\beta}} \frac{\zeta}{v}$

(A.3), (A.10) and (A.12) yield a solution for steady-state health expenditure:

$\bar{x}=\left[\frac{\beta \zeta(1-\alpha)}{\rho-n+v}\left(\frac{\alpha}{\rho+\delta}\right)^{\frac{\alpha}{1-\alpha}}\right]^{\frac{1}{1-\beta}}$

Equations (A.9), (A.12) and the production function yield 
$\bar{k}=\left(\frac{\rho+\delta}{\alpha}\right)^{\frac{-1}{1-\alpha}} \bar{h}$

From production function and equation (A.9), it follows that

$\bar{y}=\left(\frac{\alpha}{\rho+\delta}\right)^{\frac{\alpha}{1-\alpha}} \bar{h}$

We obtain the steady-state value of consumption and for $\lambda$ from equations (A.2) and (A.6'):

$\bar{c}=\left\{\frac{\rho+\delta}{\alpha}-(n+\delta)-\left(\frac{\rho+\delta}{\alpha} \frac{(1-\alpha) v \beta}{\rho-n+v}\right)\right\} \bar{k}$

$\bar{\lambda}=\left\{\bar{k}^{\alpha} \bar{h}^{1-\alpha}-\bar{x}-(n+\delta) \bar{k}\right\}^{-\theta}$

Finally, (A.5') or (A.11) yield

$\bar{\mu}=\bar{\lambda} \frac{(1-\alpha)\left(\frac{\rho+\delta}{\alpha}\right)^{\frac{-\alpha}{1-\alpha}}}{v-n+\rho}$

\section{Appendix B}

The linearized form of the canonical system is

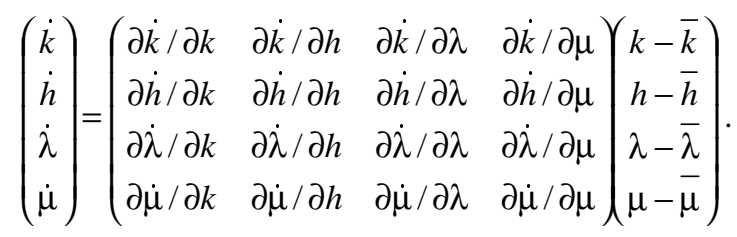

\section{Theorem. ${ }^{17}$}

If the matrix $J$ of the differential equations system given above, abbreviated as $\dot{z}=J z$, has 2 eigenvalues with negative real parts and 2 eigen-values with positive real parts, then there will be a 2dimensional stable manifold in the vicinity of the origin such that solutions, which start in this manifold, will converge to the origin.

${ }^{17}$ We reproduce theorems 5.3 and 5.4 from Feichtinger and Hartl (1986) in the form that we apply it. It is more general. 
Define ' $L$ ' as the sum of the principal minors of order 2.

\section{Theorem.}

The conditions $L<0$ and $0<$ det $J \leq L^{2} / 4$ are necessary and sufficient to get 2 negative and 2 positive real eigen-values of $J$.

To get a simplified version of $\mathrm{L}$, the following simplifying relations (B1-5) can be used ${ }^{18}$

$$
\begin{aligned}
& \frac{\partial \dot{k}}{\partial k}+\frac{\partial \dot{\lambda}}{\partial \lambda}=\left[\alpha\left(\frac{k}{h}\right)^{\alpha-1}-(n+\delta)\right]+\left[\rho-\alpha\left(\frac{k}{h}\right)^{\alpha-1}+\delta\right]=\rho-n \\
& \frac{\partial \dot{h}}{\partial h}+\frac{\partial \dot{\mu}}{\partial \mu}=-v+(v-n+\rho)=\rho-n
\end{aligned}
$$

Note that the right-hand side values of (B.1) and (B.2) are identical.

$$
\begin{aligned}
& \frac{\partial \dot{h}}{\partial k}+\frac{\partial \dot{\lambda}}{\partial \mu}=0+0=\frac{\partial \dot{k}}{\partial h}+\frac{\partial \dot{\mu}}{\partial \lambda}=(1-\alpha)\left(\frac{k}{h}\right)^{\alpha}-(1-\alpha)\left(\frac{k}{h}\right)^{\alpha}=0 \\
& \frac{\partial \dot{\lambda}}{\partial h}=\frac{\partial \dot{\mu}}{\partial k}=-\bar{\lambda}(1-\alpha) \alpha\left(\frac{k}{h}\right)^{\alpha-1} \bar{h}^{-1} \\
& \frac{\partial \dot{k}}{\partial \mu}=\frac{\partial \dot{h}}{\partial \lambda}=\frac{-1}{1-\beta}\left[\frac{\bar{\lambda}}{\bar{\mu} \zeta \beta}\right]^{\frac{-1}{1-\beta}}--1
\end{aligned}
$$

Using these relations the sum of principal minors of order 2 can be written as ${ }^{19}$

$$
\begin{aligned}
& L=\left|\begin{array}{ll}
\frac{\partial \dot{k}}{\partial k} & \frac{\partial \dot{k}}{\partial \lambda} \\
\frac{\partial \dot{\lambda}}{\partial k} & \frac{\partial \dot{\lambda}}{\partial \lambda}
\end{array}\right|+\left|\begin{array}{ll}
\frac{\partial \dot{h}}{\partial h} & \frac{\partial \dot{h}}{\partial \mu} \\
\frac{\partial \dot{\mu}}{\partial h} & \frac{\partial \dot{\mu}}{\partial \mu}
\end{array}\right|+2\left|\begin{array}{ll}
\frac{\partial \dot{k}}{\partial h} & \frac{\partial \dot{k}}{\partial \mu} \\
\frac{\partial \dot{\lambda}}{\partial h} & \frac{\partial \dot{\lambda}}{\partial \mu}
\end{array}\right| \equiv|A|+|B|+2|C| \\
& =[\frac{\partial \dot{k}}{\partial k} \cdot 0-\underbrace{\frac{\partial \dot{\lambda}}{\partial k} \frac{\partial \dot{k}}{\partial \lambda}}_{+,+}]+[\underbrace{(-v)(v-n+\rho)}_{-,+}-\underbrace{\frac{\partial \dot{\mu}}{\partial h} \frac{\partial \dot{h}}{\partial \mu}}_{+,+}]+2[\frac{\partial \dot{k}}{\partial h} \cdot 0-\underbrace{\frac{\partial \dot{\lambda}}{\partial h} \frac{\partial \dot{k}}{\partial \mu}}_{-,-}]<0
\end{aligned}
$$

\footnotetext{
${ }^{18}$ They correspond to equations 5.34 in Feichtinger and Hartl (1986). Except for details in the middle part of the equations they hold in general for canonical systems with $\rho-n$ as the discount factor.
} 
The signs of the partial derivatives can be checked looking at the canonical system.

Exchanging the second and third column of $J$ and also exchanging the second and third row of $\mathrm{J}$, using the definitions of the matrices A, B and C given in (B.6) allows writing $\mathrm{J}$ as a partitioned matrix:

$$
\begin{aligned}
& J=\left(\begin{array}{ll}
A & C \\
D & B
\end{array}\right) \text { with } \\
& D \equiv\left(\begin{array}{cc}
0 & \frac{\partial \dot{k}}{\partial \mu} \\
\frac{\partial \dot{\lambda}}{\partial h} & -\frac{\partial \dot{k}}{\partial h}
\end{array}\right)
\end{aligned}
$$

$\mathrm{D}$ is very similar to $\mathrm{C}$ and has the same determinant. It is well known that for this partitioned matrix we can write the determinant as

$$
|J|=|B|\left|A-C B^{-1} D\right|=|A|\left|B-D A^{-1} C\right|
$$

Unfortunately, three of the elements of B have the same sign as $\mathrm{DA}^{-1} \mathrm{C}$. Therefore we can proceed only numerically. We do so in appendix $\mathrm{C}$.

\footnotetext{
${ }^{19}$ See Feichtinger and Hartl (1986) equation (5.37).
} 


\section{References}

Barro, Robert J. (1991), "Economic Growth in a Cross-section of Countries", Quarterly Journal of Economics, Vol.106, pp. 407-443.

Barro, Robert J. and Xavier Sala-i-Martin (1992), “Convergence”, Journal of Political Economy, Vol. 100, pp. 223-251.

Barro, Robert J. and Xavier Sala-i-Martin (1995). Economic Growth, McGraw-Hill, Inc. U.S.A.

Bloom, David.E. and Ajay S. Mahal (1995), "Does the AIDS Epidemic Really Threaten Economic Growth”, NBER Working Paper No. 5148.

Cass, David (1965). "Optimum Growth in an Aggregative Model of Capital Accumulation", Review of Economic Studies, Vol. (32), pp. 233-40.

Carrin, G. and C. Politi (1995). "Exploring the Health Impact of Economic Growth, Poverty Reduction and Public Health Expenditure”, Tijdschrift Voor Economie en Management, Vol. 40, pp. 227-246.

Cuddington, John T.; Hancock, John D. and Rogers, Carol Ann. (1994). "A Dynamic Aggregative Model of the AIDS Epidemic With Possible Policy Interventions", Journal of Policy Modelling, Vol. 16(5), pp. 473-496.

Feichtinger, G. and R.F. Hartl (1986), Optimal Kontrolle ökonomischer Prozesse, de Gruyter, Berlin, New York.

Inada, Ken-Ichi. (1963). "On a Two-Sector Model of Economic Growth: Comments and a Generalisation”, Review of Economic Studies, Vol. 30, pp. 119-27.

Knowles, Stephen and Owen, Dorian P., (1995). "Health Capital and Cross-country Variation in per Capita in the Mankiw-Romer-Weil Model”, Economics Letters, Vol. 48 (223), pp. 99-106.

Knowles, Stephen and Owen, Dorian P., (1997). "Education and Health in an Effective-Labour Empirical Growth Model”, The Economic Record, Vol. 73 (223), pp. 314-28.

Koopmans, Tjalling (1965). "On the Concept of Optimal Economic Growth", in The Econometric Approach to Development Planning, Amsterdam, North Holland. 
Lucas, R.E.Jr. (1988). "On the Mechanics of Economic Development", Journal of Monetary Economics, Vol. 22, pp. 3-42.

Mankiw, N.G., D. Romer and D.N. Weil, (1992). "A contribution to the Empirics of Economic Growth”, Quarterly Journal of Economics, Vol. 107, pp. 407-437.

Mazumdar, Krishna. (1996). "An Analysis of Causal Flow Between Social Development and Economic Growth: The Social Development Index", American Journal of Economics and Sociology, Vol. 55 (3), pp. 361-383.

Mushkin, S.J. (1962). "Health as an Investment”, Journal of Political Economy, Vol. 70, pp. S129S157.

OECD (1999). OECD Health data 1999: A Comparative Analysis of 29 Countries, Paris.

Schultz, T.W. (1961). "Investment in Human Capital”, American Economic Review, Vol. 51, pp. 1-17.

Turnovsky, S.J. (1981), The optimal intertemporal choice of inflation and unemployment, Journal of Economic dynamics and control, 3, 357-84.

Yetkiner, I. H., J. Muysken, and T. Ziesemer (1999) “A Short Essay on Health and Growth”, Mimeo, University of Groningen.

van Zon, Adriaan H. and Muysken, Joan (1997), "Health, Education and Endogenous Growth", MERIT, Working Paper No: 2/97-009, Maastricht, The Netherlands. 